\title{
What strategy central nervous system uses to perform a movement balanced? Biomechatronical simulation of human lifting
}

\author{
Ali Leylavi Shoushtari* \\ Department of Computer Engineering, Shoushtar Branch, Islamic Azad University, Shoushtar, Iran
}

\begin{abstract}
How does the central nervous system control the body posture during various tasks despite a redundancy? It's a well-known question existed in such fields of study as biomechanics and bioengineering. Some techniques based on muscle and torques synergies are presented to study the function which Central Nervous System uses to addresses the kinetic redundancy in musculoskeletal system. The human body with its whole numerous joints considered as a hyper redundant structure which caused to be seemed that it is impossible for CNS to control and signal such system. To solve the kinematic redundancy in previous studies it is hypothesize that CNS functions as an optimizer, such of that are the task-based algorithms which search to find optimal solution for each specific task. In this research a new objective function based on ankle torques during movement is implemented to guarantee the stability of motion. A 2D 5DOF biomechatronical model of human body is subjected to lifting task simulation. The simulation process implements inverse dynamics as major constraint to consider the dynamics of motion for predicted postures. In the previous optimization-based techniques which are used to simulate the human movements, the motion stability was guaranteed by a nonlinear inequality constraint which restricts the total moment arm of the links to an upper and lower boundary. In this method, there is no need to use this constraint. The results show that the simulated postures are normal and the predicted motion is performed completely balanced.
\end{abstract}

Keywords: CNS, balanced movement, inverse dynamics, predictive dynamics, human motion simulation

\section{Introduction}

Multibody dynamic model of human body is used in an extensive area of research contains robotics, biomechatronics and biomedical engineering since it can provide an approach to find some variables that are not possible to be measured like: torques and internal forces of joints, stress exerted to joint's soft tissues. These mechanical parameters are so important to understand joint disease initiation and progression, like osteoarthritis $[4,17]$. In addition to pathological

\footnotetext{
*Corresponding author: Ali Leylavi Shoushtari, Department of Computer Engineering, Shoushtar Branch, Islamic Azad University, Shoushtar, Iran. E-mail: st_a_leilavi@azad.ac.ir.
}

aspects, the graphical simulation purposes are one of the major applications of human body modeling.

In order to know how the body postures vary during different movements, a dynamic model of the whole human body is applied in the movement simulation process. The motion simulation algorithms are used to analyze human movements; these are commonly implemented for athletics in order to improve the performance of the motion and to prevent injuries due to the incorrect movements [11]. Some abnormalities occurred in parts of the musculoskeletal system which are resulted in inaccurate function of the muscle activation and control $[16,18,29]$. Therefore, it is very important to know how the central nervous system 
(CNS) controls the variation of body posture during different tasks.

There is a need to large number of degrees of freedom to model human body more exactly and accurately. From a designer point of view, the redundancy is a profit because it makes such systems maneuverable, while from a analyzer view point it is a problem. A system which is designed with numerous degrees of freedom is able to do complicated movements skillfully and this is the benefit of redundancy and the problem is difficultness to analysis the motion of this system. On the other hand, the multiplicity of the joint space variables (DOFs) causes the model maneuverable and creates the redundancy problem too. We face the redundancy problem when the number of DOFs is more than needed to perform a task. This problem is also existed in robotics which is classified based on kinematics, dynamics and control types $[8,20-22]$. The model of human body usually implemented in computational algorithm by considering a large number of DOFs for motion simulation purposes. Optimization-based approaches are good solutions to overcome with the redundancy problem [23]. Some of these techniques are applied to robotic manipulator models with redundant DOFs [6, 9, 22, 26]. These methods is used for predict the posture of humanoid robots during lifting task based on the algorithm which CNS uses to controls the human postures [15]. Optimization-based solutions are completely suitable to solve the problem with a large number of variables, because such methods use a small amount of data as the inputs to result a large number of variables as the output set. The inputs contain two sets of constraints which are considered in motion simulation process: (1) the constraints which are calculated by using dynamic equations of motion, and (2) sets of algebraic equations which define the kinematics of the system.

CNS controls the configuration of body segments to perform movement with balance such as walking, running, sitting and lifting. These daily living activities are good examples of the tasks which are performed completely balanced involuntarily. Actually CNS uses an unknown algorithm to control such tasks unconsciously. Optimization-based simulation methods have an analogous performance with CNS function causing balanced movements [19]. These approaches use an objective function description subjected to be minimized; it is hypothesis as the function of CNS. On the other hand, to simulate a motion as similar as what human does, it is assumed that optimization approach chooses the set of answers which minimizes the objective function which also is considered by CNS to be minimized too.

However there are different definitions of stability in medicine and engineering, but they have the same meaning. In engineering, the stability has a mathematical definition commonly described by Lyapunov laws. According to such definition, a stable system has a minus energy performance which is called "Lyapunov Candidate Function" but the criteria of stability in medicine defines as pain threshold. A stable system in engineering is the one that has the convergence and bounded outputs. Based on this statement, the instable system has divergence outputs. In medicine, crossing the pain threshold results undesirable motions that CNS can't controls musculoskeletal system to perform the tasks appropriately. In this optimization approaches, the stability of the motion is guaranteed by using constraints which restrict the total moment arms (TMA) of segments of human body between horizontal position of heel and toe $[10,24]$. In fact, this constraint prevents of figurate the postures which will cause the falling forward and backward. In this research the optimization-based algorithm named predictive dynamics [27] with the objective function consisted of ankle torque squares summation during lifting time are used. In such approach, the inverse dynamics, limitation of joints torque and joints ranges of motion are used as constraints to construct the motion similar to real lifting movement. By and large, in this algorithm two kinds of constraints are used: 1- The constraints which shape the simulated motion similar to lifting movement consisted of two classes of constraint: a) Kinematical governing constraints such ones which determine initial and final position of box, body collision avoidance and constraints which guarantee the moving up motion of the box. b) Dynamic governing constrains which actually is a set of differential equations of motion in inverse dynamic form implemented as equality constraint to govern the dynamics of the motion in the simulation process. 2- The second type named "bounder constraint" determine of the variation of the designed variables. This classification is illustrated in Fig. 1.

Ankle torque amplitude is considered as the index of boundary stability and the optimization algorithm tries to minimize the integral of ankle torque squares during the lifting time. A five DOF biomechatronical model of the whole human body represented in part 2 is designed in Denavit-Hartenberg framework. 


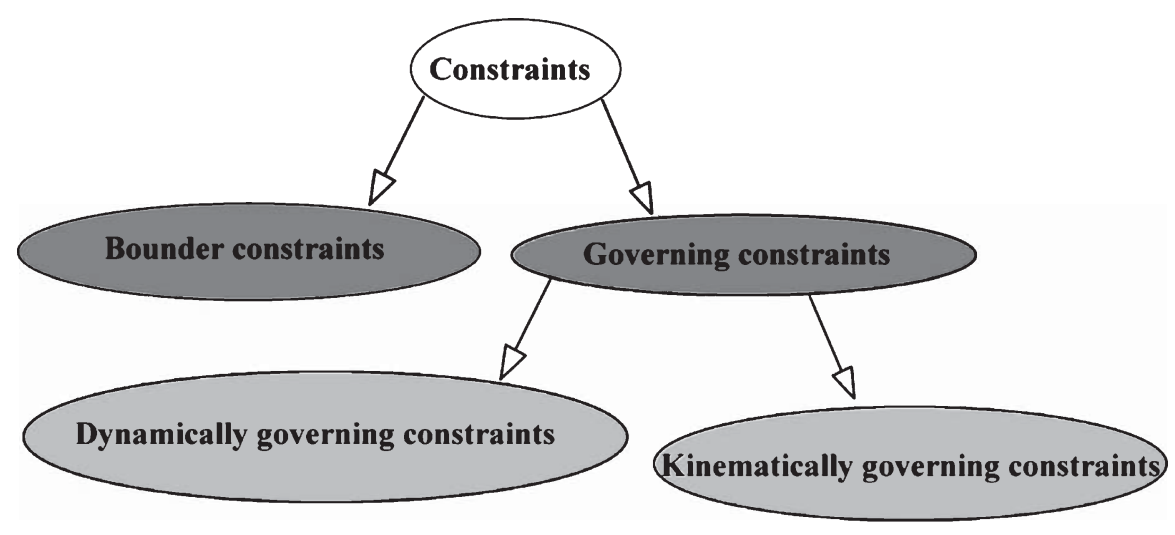

Fig. 1. Constraints classification.

The dynamics of the motion formulated based on the Lagrangian method which results the equation of the motion in inverse dynamics form. In part 3 the simulation process is described and parts 4 and 5 present results of predicted motion and conclusion remarks respectively.

\section{Modeling}

A planar model with 5DOF in sagittal plane is utilized to represent kinematics and dynamic of human body. All the limbs as shank, thigh, spine, arm and forearm are subjected to the modeling ore considered as rigid bars with mass points at the center of the mass of each link which are named $1_{1}, 1_{2}, 1_{3}, 1_{4}$, $1_{5}$ respectively. For human major joints: ankle, knee, hip, shoulder and elbow are considered joint angles to figurate human body posture and represented by the names as $q_{1}, q_{2}, q_{3}, q_{4}, q_{5}$ respectively. The box is assumed to be jointed at the wrist with constant horizontal orientation. Forward kinematics of this open kinematic chain which represents Cartesian position of the end-effector (wrist) according to the joints angles is calculated based on D-H method [12].

\subsection{Forward kinematics}

The kinematical governing constraints consist of sets of algebraic equations are obtained from forward kinematics of mentioned model. To calculate the forward kinematics of an open kinematic chain, first allocate the coordinates should be allocated at each

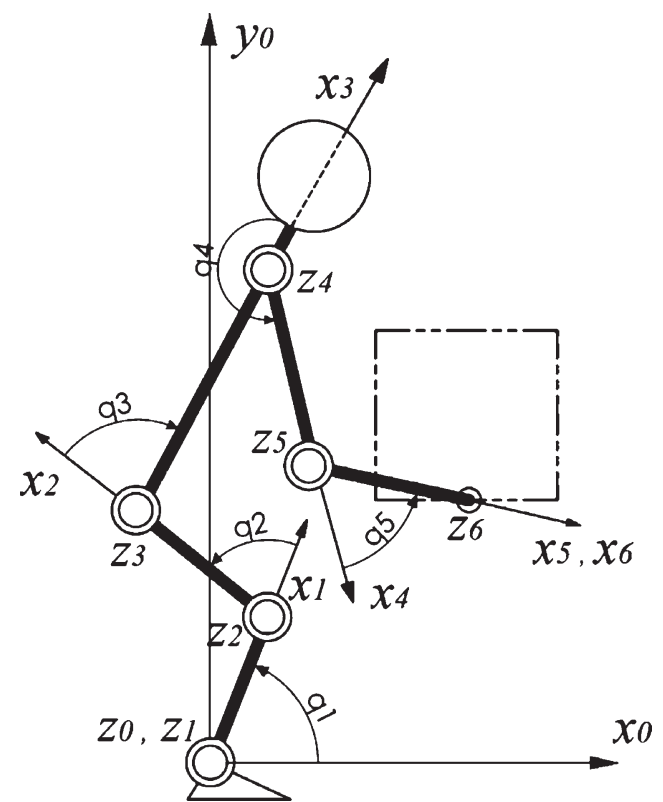

Fig. 2. 5DOF model of human body with coordination systems attached to each link. $q_{1}, q_{2}, q_{3}, q_{4}, q_{5}$ shows the angular position of ankle, knee, hip, shoulder, and elbow respectively.

link, then the relational rotation of each adjacent coordinate system is calculated, and finally the forward kinematics will obtains by post multiply the translational matrixes. Biomechatronical model of human body with coordination systems attached to limbs illustrated by Fig. 2 .

The $\left(x_{0}, y_{0}\right)$ is the global coordination system allocated to the ankle joint. The D-H parameters related to the coordination systems of the presented model are shown in Table 1. 
Table 1

D-H parameters of the human body model which is presented in Fig. 2

\begin{tabular}{llrccc}
\hline${ }_{\mathrm{i}-1} \mathrm{~T}$ & $\mathrm{I}$ & $\alpha_{\mathrm{i}-1}$ & $\mathrm{a}_{\mathrm{i}-1}$ & $\mathrm{~d}_{\mathrm{i}}$ & $\theta_{\mathrm{i}}$ \\
\hline${ }_{0}^{\mathrm{i}} \mathrm{T}$ & 1 & $0^{\circ}$ & 0 & 0 & $\mathrm{q}_{1}$ \\
$1 \mathrm{~T}$ & 2 & $0^{\circ}$ & $1_{1}$ & 0 & $\mathrm{q}_{2}$ \\
${ }_{2}^{1} \mathrm{~T}$ & 3 & $180^{\circ}$ & $1_{2}$ & 0 & $\mathrm{q}_{3}$ \\
${ }_{3}^{2} \mathrm{~T}$ & 4 & $180^{\circ}$ & $1_{3}$ & 0 & $\mathrm{q}_{4}$ \\
${ }_{4}^{3} \mathrm{~T}$ & 5 & $0^{\circ}$ & $1_{4}$ & 0 & $\mathrm{q}_{5}$ \\
${ }_{5}^{4} \mathrm{~T}$ & 6 & $0^{\circ}$ & $1_{5}$ & 0 & 0 \\
${ }_{6}^{5} \mathrm{~T}$ & & & & & \\
\hline
\end{tabular}

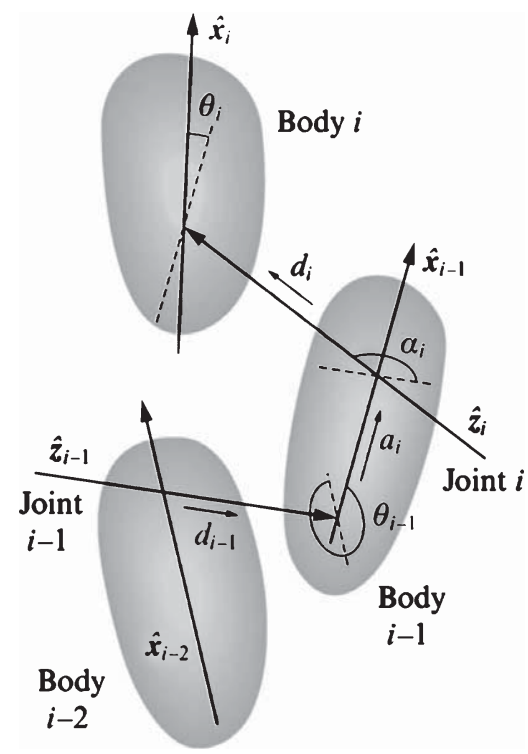

Fig. 3. Numbering adjacent joints and bodies to obtain D-H parameters [25].

Figure 3 shows how the D-H parameters are named and chosen for the two adjacent links. These parameters are: $\alpha_{i-1}, a_{i-1}, d_{i}, \theta_{i}$ which are used to calculate the translational matrix ${ }_{i}^{i-1} T$ related to each two adjacent coordination systems $i-1$ and $i$.

D-H parameters help to calculate the translational matrixes related to the adjacent coordinates calculated as (1):

$$
{ }^{i-1} T=\left[\begin{array}{cccc}
C \theta_{i} & -S \theta_{i} & 0 & a_{i} \\
S \theta_{i} C \alpha_{i} & C \theta_{i} C \alpha_{i} & -S \alpha_{i} & -S \alpha_{i} d_{i} \\
S \theta_{i} S \alpha_{i} & C \theta_{i} S \alpha_{i} & C \alpha_{i} & C \alpha_{i} d_{i} \\
0 & 0 & 0 & 1
\end{array}\right]
$$

In (1) $C \theta_{i}$ and $S \theta_{i}$ represent $\cos \left(\theta_{i}\right)$ and $\sin \left(\theta_{i}\right)$, and $C \alpha_{i}, S \alpha_{i}$ are the symbol of $\cos \left(\alpha_{i}\right)$ and $\sin \left(\alpha_{i}\right)$. Finally, by using the transformation matrix ${ }_{i}^{i-1} T$ the forward kinematics are calculated as ${ }_{6}^{0} T$ matrix which inter- prets the motion of the wrist in global coordination system connected to the ground as (2). The details of the transformational matrixes are presented in appendix.

$$
{ }_{6}^{0} T={ }_{1}^{0} T \cdot{ }_{2}^{1} T \cdot{ }_{3}^{2} T \cdot{ }_{4}^{3} T \cdot{ }_{5}^{4} T \cdot{ }_{6}^{5} T
$$

\subsection{Inverse dynamics}

The whole human body commonly models as open kinematics chain as like as robot manipulators as mentioned before [13,14], so the method used to model the dynamics of the motion of this kinematical chain is like ones used for the robotic manipulators. In this approach the Lagrangian of system is calculates, then by extermizing the integral of the lagrangian of the system, the equations which govern the dynamics of motion will be obtained. The kinetic energy of the model which was presented before [28] is defines as (3).

$$
\begin{aligned}
& K=\frac{1}{2} \dot{q}^{T} D(q) \dot{q} \\
& D(q)=\sum_{i=1}^{5}\left(m_{i} \mathrm{~J}_{\mathrm{V} c_{i}}{ }^{T} \mathrm{~J}_{\mathrm{V} c_{i}}+J_{\omega_{i}}{ }^{T} R_{i}^{0} I_{i} R_{i}^{0}\right)
\end{aligned}
$$

In Equation (3) $\dot{q}$ is $5 \times 1$ vector of the angular velocities of the joints, and $\dot{q}^{T}$ is the transpose matrix of $\dot{q}$, $D(q)$ is $5 \times 5$ matrix related to the mass and inertial properties of the model. All of the mass and inertial properties, length of and COM the links related to the subjected model are presented in Table 3 in the appendix. $J_{V c_{i}}, J_{\omega i}$ are $3 \times 5$ Jacobin matrix which translates the linear and angular velocities of COM of $i$ 'th link to the universal coordinate system respectively. $R_{i}^{o}$ is the rotational matrix transformation which interprets the orientation of the $i$ 'th links from its coordinate to the ground coordination. $m_{i}$ is the mass of $i$ 'th link. By considering $g$ as gravitational acceleration vector, and $r_{c_{i}}$ as the height of the COM of $i$ 'th link respect to the ankle position, potential energy of the system is defines as bellow:

$$
V=\sum_{i=1}^{5}\left(m_{i} g^{T} r_{c_{i}}\right)
$$

The functional of the system's energy which is called Lagrangian, is calculated as (6).

$$
L=K-V=\frac{1}{2} \dot{q}^{T} D(q) \dot{q}-\sum_{i=1}^{5}\left(m_{i} g^{T} r_{c_{i}}\right)
$$


Table 2

Parameters and their values of lifting task

\begin{tabular}{ll}
\hline Lifting parameters & \multicolumn{1}{c}{ Values } \\
\hline Box depth & $0.370(\mathrm{~m})$ \\
Box height & $0.365(\mathrm{~m})$ \\
Box weight & $9(\mathrm{~kg})$ \\
Initial height & $0.365(\mathrm{~m})$ \\
Final height & $1.37(\mathrm{~m})$ \\
Initial horizontal position & $0.490(\mathrm{~m})$ \\
Final horizontal position & $0.460(\mathrm{~m})$ \\
Lifting time duration & $1.2(\mathrm{sec})$ \\
\hline
\end{tabular}

Body segments parameters of biomechatronical model presented in Fig. 1 are as the following:

Table 3

Parameters of body segments, $l_{6}$ is the symbol of link which demonstrate the head. The $l_{1}$ to $l_{5}$ are illustrated in Fig. 1

\begin{tabular}{lcccc}
\hline Segments & Length $(\mathrm{m})$ & Mass $(\mathrm{Kgr})$ & $\mathrm{COM}(\mathrm{m})$ & Inertia $\left(\mathrm{N} . m_{2}\right)$ \\
\hline$l_{1}$ & 0.50 & 9.68 & 0.147 & 0.183 \\
$l_{2}$ & 0.46 & 33.48 & 0.209 & 0.505 \\
$l_{3}$ & 0.66 & 41.65 & 0.360 & 0.325 \\
$l_{4}$ & 0.32 & 6.36 & 0.144 & 0.070 \\
$l_{5}$ & 0.30 & 4.80 & 0.130 & 0.035 \\
$l_{6}$ & 0.12 & 6.00 & 0.058 & 0.009 \\
\hline
\end{tabular}

A functional of system's energy named $E$ is defined as the integral of system Lagrangian during the lifting time interval [0 T], as follow:

$$
\begin{aligned}
& E=\int_{t=0}^{T} L(q, \dot{q}, t) d t \\
& \frac{d}{d t}\left(\frac{\partial L}{\partial \dot{q}}\right)-\frac{\partial L}{\partial q}=\Gamma
\end{aligned}
$$

Euler-Lagrange formulation represented in (8)is an analytical method to extremize the integral of $E$ to obtain equations of the motion. In (8) $\Gamma$ is a generalized joints torque vector inserted to this formulation as an external generalized force vector. Finally the general form of the motion equations will be obtained as (9).

$$
D(q) \ddot{q}+C(q, \dot{q}) \dot{q}+V(q)=\Gamma
$$

In (9) $C(q, \dot{q})$ is a term related to the centrifugal and coriolis forces and $V(q)$ is the gravitational forces vector. These terms are calculated as follow:

$$
C(q, \dot{q})=\dot{D}(q)-\frac{1}{2} \dot{q}^{T}\left(\frac{\partial D(q)}{\partial q}\right)
$$

$$
V(q)=\left(\frac{\partial V}{\partial q}\right)^{T}
$$

The generalized joint torque represented in (9) is divided into two parts: 1 . The torques resulted in muscle forces and 2 . The torques due to the load of the box which are exerted on the wrist. These kinds are obtained as (12):

$$
\Gamma=\tau_{\text {muscle }}-\tau_{\text {box }} ; \quad \tau_{\text {box }}=J^{T}\left(m_{\text {box }} g^{T}\right)
$$

In (12) $J^{T}$ is the transpose of the Jacobian matrix which projects the box load to the joints. $m_{b o x}$ is the box mass and $g^{T}$ is the transpose of the gravity acceleration vector.

\section{Optimization-based simulation}

In this paper the lifting movement simulation is considered as the optimization procedure which CNS does as well. In this problem an objective function is subjected to be minimized with some constraints which limit the range of allowable motions to a feasible range to construct the motion realistically. In other words, it is assumed that CNS tries to minimize a particular function value to perform each task, and musculoskeletal system imposes some constraints on the motion, too. This assumption is implemented in some biomechanical researches to simulate the CNS action to predict the muscle forces and limb motions [1-3, 19]. The predictive dynamics is a novel approach used for the motion simulation [27, 28]; it implements inverse dynamic equations as a major constraint to model the motion's dynamics in the simulation process. The joints torques and angles are the designed variables, so by using this method the joint angles and torques could obtain as outputs, according to the task parameters which are used as inputs. Profiles of joint torques and angles are subjected as optimization variables. Simulation process is planned as a nonlinear constrained optimization problem. The optimization algorithm tries to minimize the objective function and satisfy the constraints, too. The equation of motion which is obtained from the previous section is written in difference form by using the finite different method. The profiles of joints angles are considered as 5 splines with the 5 control points. The first and 5th control points are maintained at the initial and final time respectively to predict the optimal initial and final postures. So, there are 25 variables related to the joints profiles and 50 variables related to the torques 

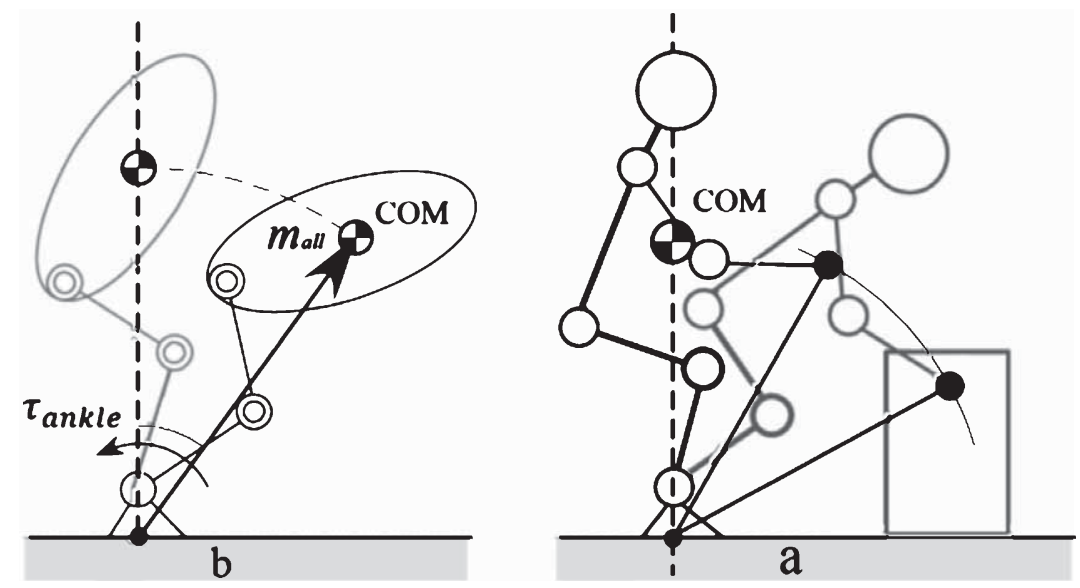

Fig. 4. a) 5DOF model of human body for lifting motion task, b) inverted pendulum equivalent model for the lifting task.

profiles of the joints for 10 time sequences. Simulation elements are described in the following sections.

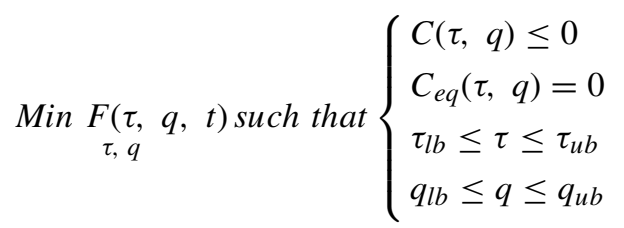

The presented structure in (13) shows the form of optimization process. In (13) $C(\tau, q)$ is inequality constraint, $C_{e q}(\tau, q)$ is equality constraints and $\tau_{l b}$ and $\tau_{u b}$ are lower and upper boundaries of limitation of joints torque respectively. $q_{l b}$ and $q_{u b}$ are lower and upper boundaries of range of motion of joints. For optimization process a computer programming code is designed by using M-file environment of MATLAB software.

\subsection{Objective function}

Considering the lifting task as a simple inverted pendulum motion, can represent good insight to analysis of motion stability. On the other hand, if the lifting motion be modeled as an inverted pendulum (Fig. 4) [5] It could be concluded that the magnitude of the pendulum joints torque has direct relation to the amount of the deviation from the stability position $\left(\theta=0^{\circ}\right)$. Therefore, a particular function which is constructed in term of the ankle torque could be used as the motion stability index. This function is the integral of the ankle torque squares in each time sequence (14). (14-A) shows a continuous form of objective function and (14-B) illustrates the digitalized form of this function. In (14-B) the $j$ is the number of time sequences which $F_{j}$ is calculated for.

$$
\begin{gathered}
F(q, \tau, t)=\int_{t=0}^{T} \tau_{\text {ankle }}^{2} d t \\
F_{j}(q, \tau)=\sum_{i=1}^{j}\left(\tau_{\text {ankle }}(i)\right)^{2} \quad \text { which } j=1,2,3 \sim 10
\end{gathered}
$$

\subsection{Constraints}

The constraints used in this research are: joints torques and angles limitations, initial and final position of the box, elevating constraint, inverse dynamics, and body collision avoidance constraint. The vertical position of the wrist (15) is a function of the joint angles $q(t)$ calculated by the forward kinematics (1):

$$
Y_{\text {wrist }}(t)=y(q(t))
$$

In each time sequence $Y_{\text {wrist }}$ should be higher than the $Y_{\text {wrist }}$ for the previous sequence:

$$
Y_{\text {wrist }}(i)>Y_{\text {wrist }}(i-1)
$$

So the elevating constraint is defined by (17).

$$
y(q(t-1))-y(q(t))<0
$$

In fact the elevating constraint is considered to guaranty the moving up motion of the box during the time sequences. The equality constraints which determine 
the initial and final position of the box are defined by (18).

$$
\left\{\begin{array}{l}
x(q(0))-x_{\text {initial }}=0 \\
y(q(0))-y_{\text {initial }}=0 \\
x(q(T))-x_{\text {final }}=0 \\
y(q(T))-y_{\text {final }}=0
\end{array}\right.
$$

$X_{\text {wrist }}(t)$ and $Y_{\text {wrist }}(t)$ are respectively horizontal and vertical positions of the wrist considered to be fixed in the box. According to the fourfold constraints set (18) should be placed at the initial position $x_{\text {initial }}$, $y_{\text {initial }}$ and final position $x_{\text {final }}, y_{\text {final }}$ of the box at $t=0$ and $t=T$ respectively. These initial and final positions of the box actually are of task parameters. Inverse dynamics constraint is expressed as below:

$$
\tau-\tau_{\text {invd }}=0 ; \quad \tau_{\text {invd }}=f(q, t)
$$

In Equation (19) $\tau$ is the joints torque vector that must be predicted, and $\tau_{i n v d}$ is the joints torque vector obtained from the inverse dynamics. Body collision avoidance which is implemented in this simulation is a

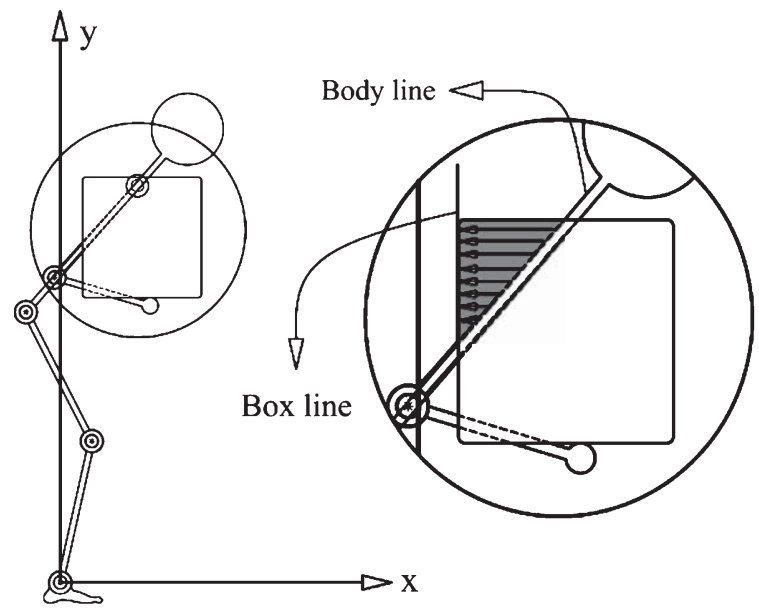

Fig. 5. Penetration of the box with the body, box line, body line and penetration aria.

systematic method used to check the penetration value of the box into the body in each iteration of the optimization process. This process is described as brief as bellow.
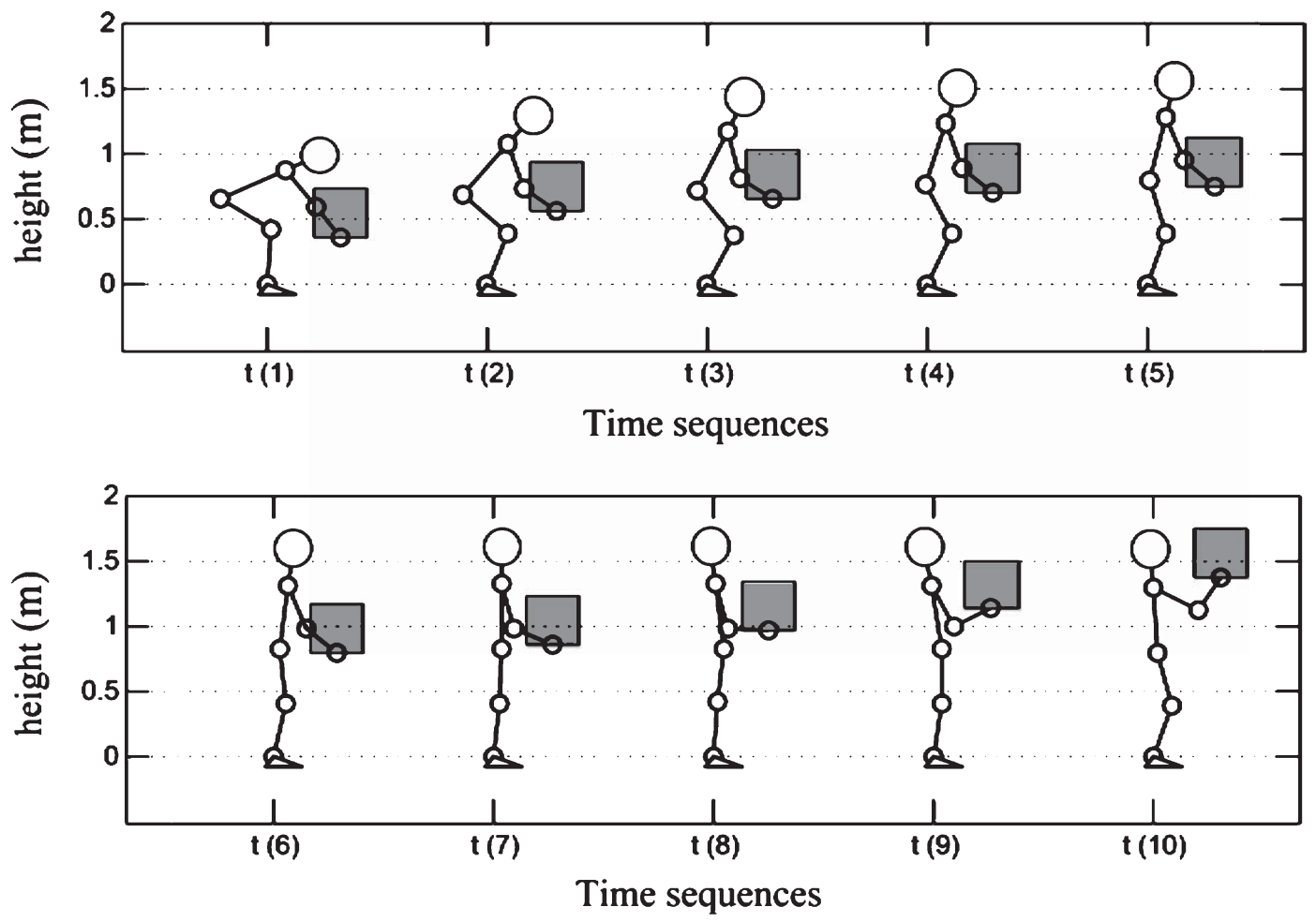

Fig. 6. The variation of human body postures during lifting task. The vertical axis shows the height in meter and the horizontal axis is time sequences. 
The collision avoidance issue is considered in the optimization process as a constraint to prevent the penetration of the box with the body. It is inequality constraint that is defined as a term of the sufficient horizontal distance $d_{x}$ that the wrist should be displaced to prevent the collision box with the body. The distance that the wrist should move to arrive at the horizontal boundary position $X_{\text {bounndary }}$ is represented by $d_{x}$, and the boundary position is a horizontal position of the place where the box edge touches the body.

$$
\begin{aligned}
& X_{\text {bounndary }-X_{\text {wrist }_{d}}<0} \\
& X_{\text {bounndary }}=X_{\text {wrist }_{p r}}+d_{x}
\end{aligned}
$$

$X_{\text {wrist }_{d}}$ is the desired horizontal position of the wrist which should be greater than $X_{\text {bounndary. }} X_{\text {wrist }_{p r}}$ is the horizontal position of the wrist obtained from the optimization algorithm in the current iteration. According to Fig. 5 the penetration value of the box in the body $d$ will calculate via the Equation (21). The values of $X_{b o d y}$ and $X_{\text {edge }}$ obtained from the body line and the box line respectively. The maximum penetration value determines the horizontal distance that the wrist should move to arrive at the boundary position. " $d$ " is the penetration index, so " $d_{x}$ " is define as the maximum value of " $d$ " (22).

$$
\begin{aligned}
& d=X_{\text {body }}-X_{\text {edge }} \\
& d_{x}=\operatorname{MAX}(d)
\end{aligned}
$$

\section{Results}

The optimization process is designed for 10 evenly distributed time sequences. By considering the 5 joints angles and torques, we have 75 variables subjected to be optimized. Inertial properties of human segments are considered as the data used previously [7]. The experimental data which used in the [28], is implemented to validate the result of simulation process. The subjected population of experiment had an average height $5^{\prime} 7^{\prime \prime}$ and the average weight of $143 \mathrm{lbs}$, the mean age of the participants was 34 years. The lifting task parameters are presented in Table 2.

The total moment arm (TMA) of the all links is calculated as (23). It is calculated from the moments related to the all links weight for each configuration of body segments in time $t . m_{\text {all }}$ is the total weight of the body and $x_{i}(t)$ is the horizontal position of center
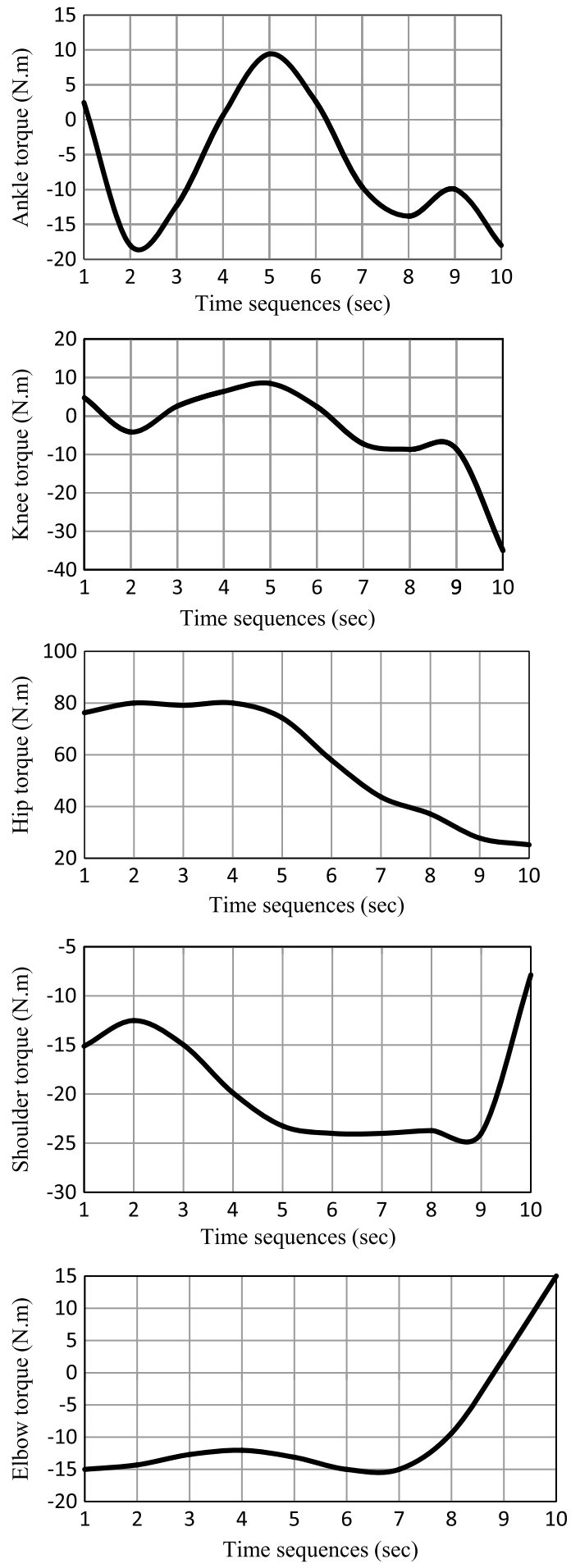

Fig. 7. Joints torque profile. 

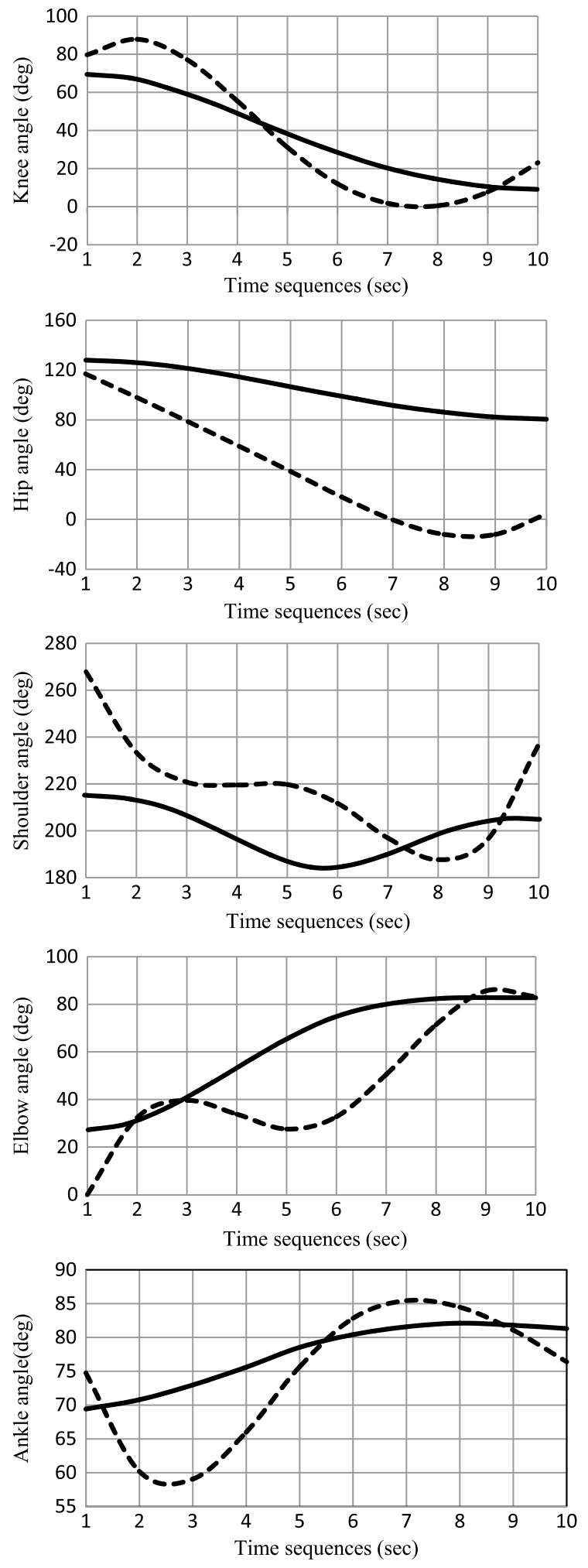

of mass of $i^{\prime}$ th links at time $t$, and $N$ is the number of links. Optimal profiles of joint angles show that how the body posture varies during the lifting task, it is illustrated in Fig. 6.

$$
T M A(t)=\frac{1}{m_{\text {all }}} \sum_{i=1}^{N} m_{i} x_{i}(t)
$$

The profiles of the joints torque profiles are shown in Fig. 7.

The predicted and experimental profile of human joints for lifting task is presented by Fig. 8. The solid lines are the predicted and the broken lines show the experimental results.

TMA which is calculated based on (22) is plotted in Fig. 9 for all the 10 time sequences. The broken lines show the upper and lower boundaries of the stable region of the motion (base of support). In fact, these are the horizontal position of the heel and toe in sagittal plane. In case of crossing from these boundaries, the human body will fall forward or backward.

\section{Discussion}

Simulation process has implemented a biomechatronical 5DOF model of the human body to simulate the lifting motion by using the unified optimization-based approach. The constraints applied to these processes, limit the range of the motions to a region that the actual human limbs could move through it. The major constraint which is named inverse dynamics exerts the dynamics of the motion to the simulation process and finally minimizing the objective function forms the shapes of the postures. The 10 time segments with 75 variables are considered in optimization process. Figure 6 shows that: (1) the posture variation is formed in a natural configuration with the extremely uprising motion of the box, (2) accurate initial and final position of the box and (3) no collision of the box with the body. Figure 9 illustrates the TMA values during the time lifting and its boundaries. According to Fig. 9 the lifting movement is performed completely balanced thanks to TMA has a value between upper and lower boundaries of stability. In other words, minimizing the summation

Fig. 8. The optimal profiles of the joints angles in comparison with experimental results. The solid and broken lines are experimental and predicted results respectively. The vertical axis are angles in degree and the horizontal axis are time sequences in $0.25 \mathrm{sec}$ scale. 


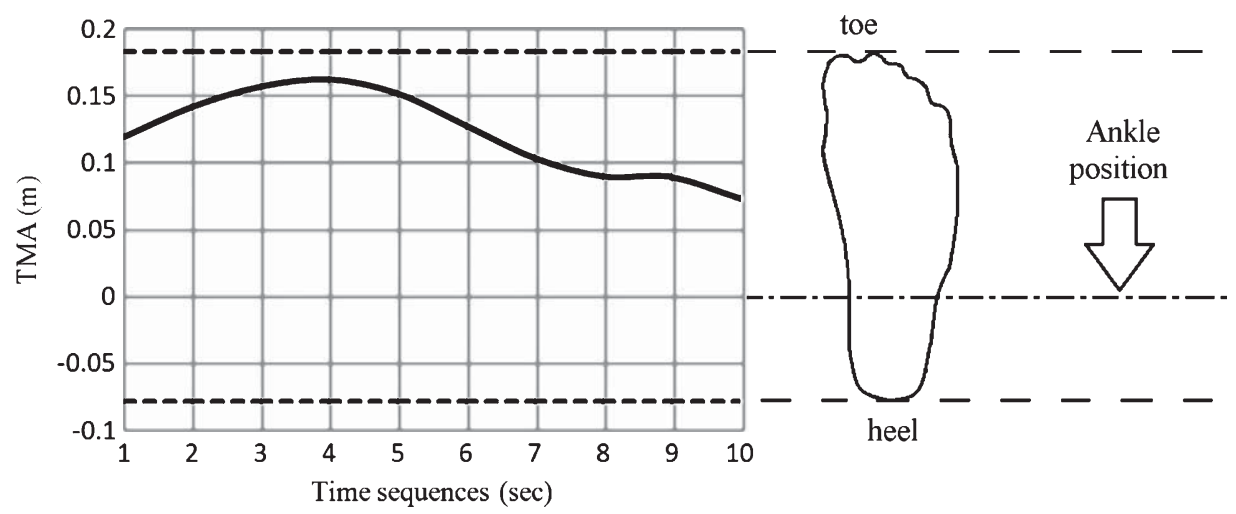

Fig. 9. TMA values during lifting time with its boundaries and relation with heel and toe. The solid lines are the calculated profile of TMA of segment of subjected model for simulated motion and broken lines are boundaries of base of support considered as margins of stability.

of squares of the ankle torque can guarantee the motion balancing.

In Fig. 8 the simulation results are compared with the experimental results due to the CNS. On the other hand, Fig. 8 illustrate a comparison between optimization approach in contrast with CNS function to control the body posture. Since this model has less number of DOFs than the actual human body, it is expected that the predicted motion profiles of the joints angles have the amplitude greater than the amplitude of experimental profiles. The predictive angles of the ankle, knee and hip joints have a good compatibility with the experimental results, and the results for the elbow are good to some extent as well. But against these joints, the shoulder's results haven't a good correlation with the experimental results. It is caused by the shoulder complex structure which needs to be modeled more exactly and considered as suitable and sufficient constraints. The implemented model is consisting of 5 serial linkages which the error of predicted angle in lower joints be added to the upper joints. Tanks to this reason the predicted angles of shoulder and elbow have not good correlation with the experimental results.

\section{Conclusion}

In sequences 6 to 10 , the profile of the ankle torque and TMA of the links have the same trends. It is concluded that in the standing posture, the ankle torque has a direct relation with TMA of the links. So by minimizing the ankle torque in standing postures the TMA also will also be minimized. This strategy can be used to guarantee the balancing of the motion in the standing postures.

According to Fig. 6 it is concluded that: in the sequences 1 to 6 , the box is lifted by the action of the joints of the lower limbs: ankle, knee and hip. In the rested sequences the lifting motion is continued by the action of the shoulder and elbow joints. On the other hand, this simulation approach is able to simulate the leg lift (squat) motion accurately. It can be used to construct the skillful movement for athletics based on the properties of their bodies which are presented in Table 2 and the lifting task parameters presented in Table 1. It can also be implemented to improve the performance of the athletic movements and the injury presentation using the algorithm to produce standard pattern for each given task.

\section{Appendix}

Transformer matrixes used to calculate forward kinematics are as below:

$$
\begin{aligned}
& { }_{1}^{0} T=\left[\begin{array}{cccc}
C \theta_{1} & -S \theta_{1} & 0 & 0 \\
S \theta_{1} & C \theta_{1} & 0 & 0 \\
0 & 0 & 1 & 0 \\
0 & 0 & 0 & 1
\end{array}\right] \\
& { }_{2}^{1} T=\left[\begin{array}{cccc}
C \theta_{2} & -S \theta_{2} & 0 & 1_{1} \\
S \theta_{2} & C \theta_{2} & 0 & 0 \\
0 & 0 & 1 & 0 \\
0 & 0 & 0 & 1
\end{array}\right]
\end{aligned}
$$




$$
\begin{aligned}
& { }_{3}^{2} T=\left[\begin{array}{cccc}
C \theta_{2} & -S \theta_{2} & 0 & 1_{2} \\
-S \theta_{2} & -C \theta_{2} & 0 & 0 \\
0 & 0 & -1 & 0 \\
0 & 0 & 0 & 1
\end{array}\right] \\
& { }_{4}^{3} T=\left[\begin{array}{cccc}
C \theta_{4} & -S \theta_{4} & 0 & 1 \\
-S \theta_{4} & -C \theta_{4} & 0 & 0 \\
0 & 0 & -1 & 0 \\
0 & 0 & 0 & 1
\end{array}\right] \\
& { }_{5}^{4} T=\left[\begin{array}{cccc}
C \theta_{5} & -S \theta_{5} & 0 & 1_{4} \\
S \theta_{5} & C \theta_{5} & 0 & 0 \\
0 & 0 & 1 & 0 \\
0 & 0 & 0 & 1
\end{array}\right] \\
& { }_{6}^{5} T=\left[\begin{array}{llll}
1 & 0 & 0 & 1 \\
0 & 1 & 0 & 0 \\
0 & 0 & 1 & 0 \\
0 & 0 & 0 & 1
\end{array}\right]
\end{aligned}
$$

\section{References}

[1] P. Abedi and A. Leylavi Shoushtari, Modeling and simulation of human-like movements for humanoid robots. In: Proceeding of ICINCO 2012-International Conference on Informatics in Control, Automation and Robotics Rome, Italy, 2 (2012), 342-346.

[2] F.C. Anderson and M.G. Pandy, Dynamic optimization of human walking, Journal of Biomechanical Engineering 123(5) (2001), 381-390.

[3] F.C. Anderson and M.G. Pandy, Static and dynamic optimization solutions for gait are practically equivalent, Journal of Biomechanics 34(2) (2001), 153-161.

[4] T.P. Andriacchi, A. Mundermann, R.L. Smith, E.J. Alexander, C.O. Dyrby and S. Koo, A framework for the in vivo pathomechanics of osteoarthritis at the knee, Annals of Biomedical Engineering 32 (2004), 447-457.

[5] H. Arisumi, J.R. Chardonnet, A. Kheddar and K. Yokoi, Dynamic Lifting Motion of Humanoid Robots. In: Proceeding of IEEE International Conference on Robotics and Automation, Rome, Italy, 2007, pp. 2661-2667.

[6] K. Belda, J. Böhm and M. Valášek, State-Space generalized predictive control for redundant parallel robots, Mechanics Based Design of Structures and Machines 31(3) (2003), 413-432.

[7] W. Blajer, K. Dziewiecki and Z. Mazur, Multibody modeling of human body for the inverse dynamics analysis of sagittal plane movements, Journal of Multibody Systems Dynamics 18(2) (2007), 217-232.

[8] S.H. Cha, T.A. Lasky and S.A. Velinsky, Kinematic redundancy resolution for serial-parallel manipulators via local optimization including joint constraints, Mechanics Based
Design of Structures and Machines 34(2) (2006), 213239.

[9] S.H. Cha, T.A. Lasky and S.A. Velinsky, KinematicallyRedundant Variations of the 3-RRR Mechanism and Local Optimization-Based Singularity voidance, Mechanics Based Design of Structures and Machines 35(1) (2007), $15-38$.

[10] C.C. Chang, D.R. Brown, D.S. Bloswick and S.M. Hsiang, Biomechanical Simulation of manual lifting using time space, Journal of Biomechanics 34(4) (2001), 527-532.

[11] E. Demircan, O. Khatob, J. Wheeler and S. Delp, Reconstruction and EMG-Informed control, simulation and analysis of human movement for athletics: Performance improvement and injury prevention, In: Proceeding of IEEE International Conference on Engineering in Medicine and Biology Society Minneapolis, MN, 2009, pp. 6534-6537.

[12] J. Denavit and R.S. Hartenberg, A kinematic notation for lower-pair mechanisms based on matrices, Transaction of ASME Journal of Applied Mechanics 22 (1955), 215221.

[13] O. Khatib, J. Warren, V.D. Sapio and L. Sentic Human like motion from physiologically-based potential energy, In: J. Lenarčič and C. Galletti, (Eds.) On Advances in Robot Kinematics. Kluwer Academic Publishers, Netherland, 2004, pp. 149-163.

[14] O. Katib, E. Demircan, V.D. Sapio, L. Sentic, T. Besier and S. Delp, Robotic-based Synthesis of human motion, Journal of Physiology-Paris 103(3) (2009), 211-219.

[15] A. Leylavi Shoushtari and P. Abedi, Realistic dynamic posture prediction of humanoid robot: Manual lifting task simulation, In: C.-Y. Su, S. Realistic and H. Liu (Eds.), Inteligent Robotics and Applications, subseries: Lecture Notes in Artificial Intelligence Vol. 7506, Berlin, (C Springer-Verlag, 2012, pp. 565-578.

[16] G. Li, K.R. Kaufman, E.Y. Chao and H.E. Rubash, Prediction of antagonistic muscle forces using inverse dynamic optimization during flexion/extension of the knee, Journal of Biomechanical Engineering 121(3) (1999).

[17] T. Miyazakai, M. Wada, H. Kawahara, M. Sato, H. Baba and S. Shimada, Dynamic load at baseline can predict radiographic disease progression in medial compartment knee osteoarthritis, Annals of Rheumatic Diseases 61 (2002), 617-622.

[18] M.A. Nussbaum and D.B. Chaffin, Lumbar muscle force using a subject-invariant 5-parameter EMG-based model, Journal of Biomechanics 31(7) (1998), 667-672.

[19] M.G. Pandy, Computer modelling and simulation of human movement, Annual Review of Biomedical Engineering 3 (2001), 245-732.

[20] K.C. Park, P.H. Chang and S. Lee, Analysis and control of redundant manipulator dynamics based on an extended operational space, Robotica 19(6) (2001), 649-662.

[21] J. Peters, M. Mistry, F. Udwadia, J. Nakanishi and S. Schaal, A unifying framework for robot control with redundant DOFs, Autonomous Robots 24(1) (2008), 1-12.

[22] B. Schafer, R. Krenn and B. Rebele, On inverse kinematics and kinetics of redundant space manipulator simulation, Journal of Computational and Applied Mechanics 4(1) (2003), 53-70.

[23] M.P.T. Silva and J.A.C. Ambrósio, Solution of redundant muscle forces in human locomotion with multibody dynamics and optimization tools, Mechanics Based Design of Structures and Machines 31(3) (2003), 381-411. 
[24] M.K. Sitoh, J.G. Chen, K. Leng and H.S. Jung, A graphical computer system for modeling the manual lifting task via biomechanical and psychophysical-biomechanical optimization approaches, Journals of Computers in Industry 21(2) (1993), 149-165.

[25] K. Waldron and J. Schmiedeler, Kinematics In: B. Siciliano and O. Khatib (Eds.) Springer Handbook of Robotics. Berlin, (C) Springer-Verlag, 2008, pp. 9-31.

[26] J. Wang, Y. Li and X. Zhao, Inverse Kinematics and Control of a 7-DOF Redundant Manipulator Based on the Closed-Loop Algorithm, International journal of Advanced Robotic Systems 7(4) (2010), 1-10.
[27] Y. Xiang, J.S. Arora, S. Rahmatalla and K. Abdel-Malek, Optimization-based dynamic human walking prediction: One step formulation, International Journal of Numerical Methods in Engineering 79(6) (2009), 667-695.

[28] Y. Xiang, J.S. Arora, S. Rahmatalla, T. Marler, R. Bhatt and K. Abdel-Malek, Human lifting simulation using a multi-objective optimization approach, Journal of Multibody Dynamics 23(4) (2010), 431-451.

[29] S.Zeinali-Davarani, H. Hemami, K. Barin and A. Shirazi-Adl, Dynamic stability of spine using stability-based optimization and spindle reflex, IEEE Transaction of Neural System Rehabilitation Engineering 16(1) (2008), 106-118. 

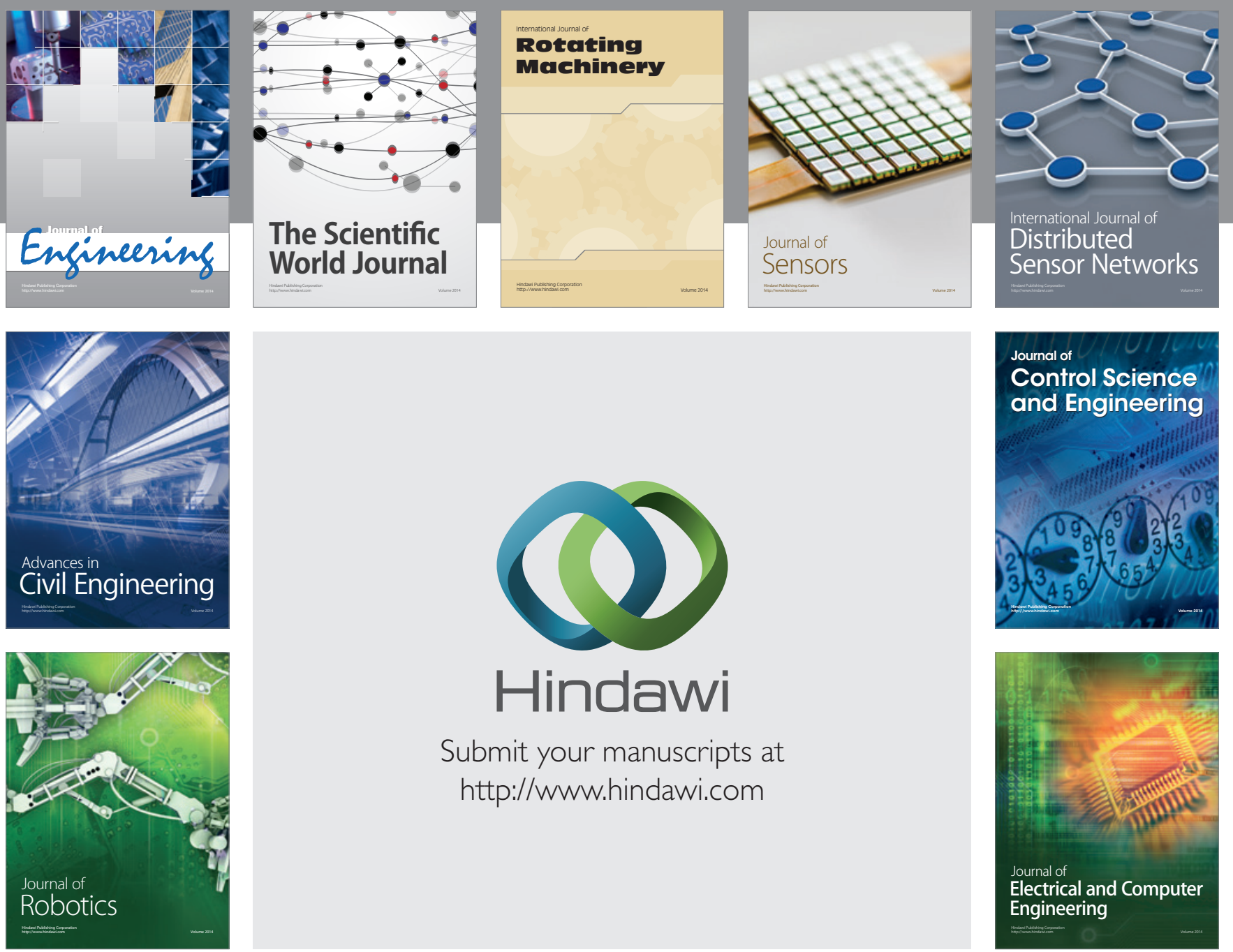

Submit your manuscripts at

http://www.hindawi.com
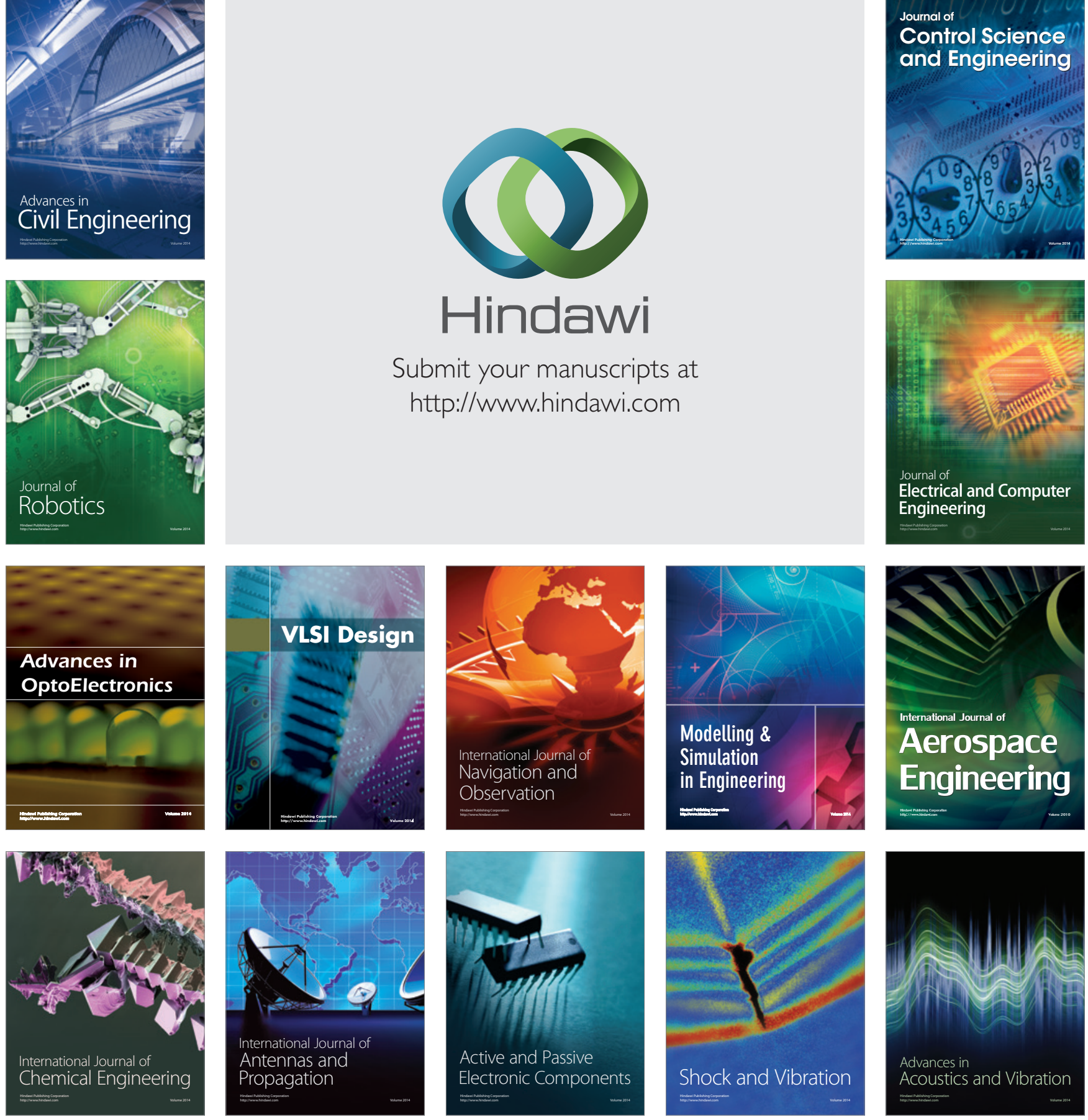